
AFTER INFLUENZA VIRUS INFECTION IN PAEDIATRIC PATIENTS

doi:10.1136/archdischild-2012-302724.0644

10 Genovese, 'S Pulitanò, ${ }^{2} \mathrm{~S}$ Crocco, ${ }^{1} \mathrm{~A}$ Mancino, ${ }^{1} \mathrm{~F}$ Tosi, ${ }^{1} \mathrm{M}$ Piastra. ${ }^{1}$ Paediatric Intensive Care Unit; ${ }^{2}$ Pediatrics, Policlinico Gemelli University Hospital, Roma, Italy

Influenza virus can cause common respiratory tract infections and rarely multiorgan system disorders, resulting in mild infection, severe respiratory disease or systemic disease and complications. CNS dysfunction, an important complication of influenza infection includes IAE, a rapid progressive encephalopathy that usually presents in the early phase of influenza infection. Because of lack of inflammation in the CNS, IAE is always named influenza-associated acute encephalopathy, which includes acute necrotizing encephalopathy (ANE), presenting with fulminant encephalopathy and characteristic brain lesions following viral infection, which pathogenesis is not fully understood, but associated with unfavourable outcome.

We present four new cases of central nervous system dysfunction subsequent to infection with Influenza Virus. All four cases had convincing evidence of preceding Influenza disease with no evidence of viable Influenza Virus in the cerebrospinal fluid. We propose that these cases represent examples of post Influenza central nervous system dysfunction. We also present a review of the literature regarding Influenza neurologic dysfunction and speculate on the underlying pathologic mechanisms.

\section{ACUTE ENCEPHALITIS COMPLICATING RUBELLA: FOUR CASE REPORTS}

doi:10.1136/archdischild-2012-302724.0645

K Lajmi, S Haddad, S Hammami, C Ben Meriem, L Ghedira, MN Gueddiche. Fattouma Bourguiba Hospital, Monastir, Tunisia

Background Rubella encephalitis is a rare complication of rubella with an incidence that has been reported to range from 1/4300 to $1 / 24000$.

Methods We report four pediatric cases of acute encephalitis complicating rubella during an epidemic of rubella between March 2011 and November 2011 to the Fattouma Bourguiba Hospital.

Results Three males and one female were included. The mean age was 9 years ( $7-12$ years), rubella vaccine have been given only for two patients. Macular rash was reported in three cases. The most clinical symptoms were: headache, fever, loss of consciousness and seizures. Serum immunoglobulin (Ig) M antibodies against rubella virus were present in all cases. In all cases cerebrospinal fluid (CSF) analysis reveals lymphocytic pleocytosis, elevated protein levels and normal glucose levels and the presence of immunoglobulin $M$ antibodies against rubella virus. Electroencephalogram (EEG) showed slow wave activity without focal or paroxysmal features in all cases and computed tomographies of the brain (CT) were normal. Mechanical ventilation was needed in 3. Acyclovir was administered intravenously for all patients given the initial concern of herpes simplex encephalitis. Favourable outcomes in all cases were reported.

Conclusion In Tunisia a revision of our vaccination program against rubella is needed. Benefits and the costs of a non-selective vaccination strategy including all children and all women of childbearing age must be weighted to prevent such a severe complication. FEBRILE CONVULSIONS

doi:10.1136/archdischild-2012-302724.0646
F Akter. East Kent University Hospital Trust, East Kent, UK

Background and Aims Febrile convulsions are the most common seizure disorder in childhood, affecting $2-5 \%$ of children between the ages of 3 and 60 months. It is a frightening and anxiety-provoking event for parent and caregivers. The initial management of this condition is often poor in rural setting in third world countries.

Aims To assess the factors affecting the initial management of children with febrile convulsions.

Methods A prospective study interviewing parents of 20 children with febrile convulsions admitted to a hospital in Dhaka, Bangladesh.

Results 19 mothers and 2 fathers of children with febrile convulsions were interviewed. Only 1 parent knew what a febrile convulsion was. $50 \%$ initially managed their children by massaging oil onto the back. $60 \%$ of the children were seen by a local religious healer. $90 \%$ of parents believed their child was possessed by an evil spirit. $50 \%$ thought their children will die. $60 \%$ of the parents had been educated up to only primary school level. $90 \%$ reported their local hospital to be 10 miles away.

Conclusions The education level, religious beliefs and location of nearest hospital significantly affected the way parents initially manage their children with febrile convulsions. It is important in third world countries that media methods such as television, radio and newspapers are used to discuss management of common conditions to avoid inappropriate treatments by parents. Leaflets should also be made available to collect from local shops and family physicians.

\section{CARDIOVASCULAR ABNORMALITIES IN CHILDREN AND ADOLESCENTS WITH TYPE 1 DIABETES MELLITUS}

doi:10.1136/archdischild-2012-302724.0647

'GC Yılmaz, 'P Boran, ${ }^{2} \mathrm{~L}$ Yılmaz, ${ }^{3} \mathrm{MA}$ Astarcioglu, ${ }^{3} \mathrm{E}$ Erturk, 'E Tutar, 'G Tokuc. 'Dr. Lutfi

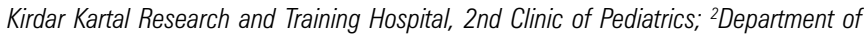
Family Medicine, Dr. Lutfi Kirdar Kartal Research and Training Hospital; ${ }^{3}$ Department of Cardiology, Kosuyolu Heart and Research Hospital, Istanbul, Turkey

Background and Aims Diabetic patients can show signs of cardiac autonomic neuropathy and diabetic cardiomyopathy.

We aimed to determine left ventricular function and cardiac autonomic nervous system function in cardiac-asymptomatic diabetic children and to examine the relationship between cardiac function abnormalities and sex, glycemic control, and diabetes duration.

Methods Left ventricular function was assessed using echocardiography. HRV was computed for each subject by means of standard 24-h time domain measures. The final data analysis was conducted on 57 diabetic patients (mean age $14.4+3.09$ years, 31 males) and 40 healthy controls (mean age $13.6+2.2$ years, 19 males).

Results No significant differences were found between patients and controls with respect to systolic and diastolic blood pressure, however heart rate measured during physical exam at rest was significantly higher in the diabetic patient group. HRV indexes were lower in the patient group but not significant. Left ventricular wall dimensions and systolic function were comparable in both groups. The A wave velocities, deceleration time and IVRT were significantly higher in the diabetic group. Tissue Doppler derived myocardial peak systolic velocity (Sm) of LV and the Tei index were normal reflecting the preserved LV systolic function. The mitral early filling velocities (Em) were significantly reduced in the diabetic group, with a consequently significant lower $\mathrm{E} / \mathrm{A}$ ratio.

Conclusions In conclusion, our diabetic patients had signs of significant left ventricular filling abnormalities. Increased heart rate and lowered HRV indexes although not significant may reflect the early evidence of cardiac autonomic nervous dysfunction. 